\title{
Epiphytic diatoms in lotic and lentic waters - diversity and representation of species complexes
}

\author{
Jan Kollár ${ }^{1}$, Markéta Fránková2, Petr Hašler ${ }^{1}$, Markéta Letáková ${ }^{1} \&$ Aloisie \\ POULÍČKOVÁ ${ }^{1}$
}

\author{
${ }^{1}$ Department of Botany, Faculty of Sciences, Palacký University Olomouc, Str. Šlechtitelů 27, CZ-783 71 Olo- \\ mouc, Czech Republic \\ ${ }^{2}$ Department of Vegetation Ecology, Institutitute of Botany, Czech Academy of Sciences, Lidická 25/27, CZ-657 \\ 20 Brno, Czech Republic
}

\begin{abstract}
Small streams and shallow ponds represent sensitive ecosystems and attached diatoms can serve as integrative indicator with fast response to environmental changes. Development of methods for ecological monitoring throughout Europe and their calibration for particular ecoregions are not finished yet and databases need to be filled by data from undersampled regions and overlooked substrates. The present study aims to explore the diversity of epiphytic diatoms in unexplored catchment areas with special attention to substrate specificity and distribution of unresolved diatom species complexes. Significant differences were found in diversity of both regions and water types (lotic/lentic). No significant differences were found in the case of substrates. CCA analysis showed significant influence of $\mathrm{pH}$, water streaming (streaming/stagnant) and Lemna substrate to species composition. Surprisingly species complexes represent the majority of epiphytic assemblages with no significant differences between lotic and lentic waters or substrates except of Lemna. The high representation of complexes does not lead automatically to reduction of overall diversity of the sample.
\end{abstract}

Key words: diatoms, epiphyton, lotic and lentic waters, species complexes

\section{INTRODUCTION}

In Europe, most shallow lakes/ponds and rivers are strongly affected by human activities. The EU members in the frame of Water Framework Directive (WFD) developed standardized methods to assess the ecological status of surface waters using bioindicators. Diatoms are considered to be good indicator organisms in aquatic ecosystems (BLANCO et al. 2004, 2014). The cross-taxon congruence of six contrasting groups of organisms (vascular plants, bryophytes, fungi, diatoms, desmids and testate amoebae) in the same permanent plots were analysed in freshwater wetlands (HÁJEK et al. 2014). The main difference among different taxa corresponded clearly with body size and life span (micro versus macroorganisms), conforming the assumption of faster response of microorganisms to environmental changes. Generally, macroorganisms provide similar information, while diatoms behave most independently (HÁsEk et al. 2014). Diatoms occupy a variety of substrates in both lotic and lentic waters. Development of methods for ecological monitoring throughout Europe (KELLY et al. 2009) and their calibration for particular ecoregions are not finished yet and databases need to be filled by data from undersampled regions and less sampled substrates. Moreover there are many problems with cryptic diversity and their ecological significance (PoulíčKovÁ et al. 2008, 2014). Some diatom traditional morphospecies included in regional floras (Sellaphora pupula, Achnanthidium minutissimum, Gomhonema parvulum etc.) have long been considered cosmopolitan, ubiquitous, and morphologically highly variable taxa. However molecular methods revealed, that these diatoms are species complexes consisting of few or many species, whose identification in LM is difficult or impossible (PotAPOVA \& HAMILTON 2007; Mann et al. 2008; PoulíčKovÁ et al. 2010). The use of benthic diatoms in the context of ecological status assessment (KING et al. 2006; KELLY et al. 2007) seems to be broadly accepted, although more studies are dealing with running waters and epilithon (RIMET \& Bouchez 2012). Methodology for shallow lakes using epiphyton has been suggested quite recently (BLANCO et al. 2014). The present study aims to explore epiphytic diatoms of small ponds and streams covering main ecological gradients of Southeastern Moravia (Czech Republic). Special attention was paid to representation of diatom species complexes at different substrates and water types and its influence to epiphyton bioindication capacity. 


\section{Material And Methods}

Samples were collected in summer 2013 and 2014 in 25 ponds and 13 streams of two sampling areas. Both regions (the Svitava region and the White Carpathian Mountains) belong to the Morava River Basin. The first one - the Svitava Highland is a part of the Svitava River basin (SB) and geologically belongs to the southeastern part of the Cretaceous Table. In the area prevail mesozoic (sandstone, marstone, marlstone, claystone) and quarternary (loam, loess, gravel, sand) sedimentary rocks. Annual mean temperature is around $6{ }^{\circ} \mathrm{C}$ and annual mean precipitaion is around $600 \mathrm{~mm}$ (ToLAsz et al. 2007). Sites located in this area lay in elevation around $500 \mathrm{~m}$ a.s.l. (Fig. 1). The second area - the White Carpathian Mountains (WC) is situated on southeast of the Czech Republic (on the western margin of the Western Carpathians) along the border with Slovakia. Geological bedrock is formed by flysch belt, in which sandstone and claystone of variable calcium content alternate. Prevailing is marl, lime-rich claystone, limestone and calcareous sandstone (HÁJEK et al. 2002). Groundwaters are carbonatogenic and their dominant mineralization process is carbonate dissolution which leads to the calcium-(magnesium)-bicarbonate type of chemistry (RAPANT et al. 1996). This chemistry type supports cold water travertine (tufa) formation. Annual mean temperature is about $8^{\circ} \mathrm{C}$ and annual mean precipitaion is about $700 \mathrm{~mm}$ (Tolasz et al. 2007). Sites located in this area are situated in altitudes from $225 \mathrm{~m}$ up to $535 \mathrm{~m}$ above sea level. Basic characteristics of investigated localities are given in Table 1.

Epiphytic communities (in littoral part of ponds and/ or streaming part of the rivers) growing on submerged macrophytes Phragmites australis (CAV.) STEUd.; Poaceae (incl. Phalaris arundinacea L., Arrhenatherum elatius (L.) J. PresL et C. Presl, Poa sp., Dactylis sp., Glyceria sp.); Typha sp.; Lemna sp.; Salix sp.; Callitriche sp. and Sparganium sp. were examined.

Diatom sampling methods followed those reccommended by Kelly et al. (1998), diatom frustules were cleaned in hydrogen peroxide (TAYLOR et al. 2007) and moun- ted in Naphrax. Four hundred individuals were identified in each sample to species level using literature (KRAMmER \& Lange-Bertalot 1986; Krammer \& Lange-Bertalot 1988; Krammer \& Lange-Bertalot 1991; Krammer 2000; LANGEBertalot 2001; Krammer 2002; Krammer 2003; Krammer \& Lange-Bertalot 2004; Lange-Bertalot et al. 2011). Nomenclature has been unified following Algaebase (GuIRY \& GuIRY 2015). Species complexes selection was based on actual list of species, recent molecular literature and own experience and are summarized in Table 2, although their list can expand in near future due to molecular studies boom. Few examples of species complexes representatives are documented in Fig. 2.

Statistical analysis. Our hypothesis assumes that diatom distribution among sampling sites is influenced by measured environmental variables. Prior to main statistical analyses we disproved correlation between geographical position and environmental variables (Spearman's correlation coefficient, $\mathrm{pH}: \mathrm{r}_{\mathrm{s}}=0.587$, conductivity: $\mathrm{r}_{\mathrm{s}}=0.033$ ). Multivariate analysis using Canoco 4.5 (Ter BraAK \& Šmilauer 2002) was carried out to test relationships between identified diatoms and environmental variables $(\mathrm{pH}$, conductivity, host plant, streaming/stagnant water). First, length of the gradient was computed using Detrended correspondence analysis (DCA, detrending by segmnents, without transformation, length of the gradient on the first axis $=5.258$, speceies data explain $11.8 \%$ on the first axis). Protocol of the Canonical correspondence analysis (CCA) was carried out as follows: imported data included diatom occurrence (\%) and environmental variables ( $\mathrm{pH}$, conductivity, flowing/stagnant water, host plant), then biplot scaling focused on inter-species distances, $\log$ transformation $\left(\mathrm{Y}^{\prime}=\log \left(\mathrm{A}^{*} \mathrm{Y}+\mathrm{B}\right), \mathrm{A}=1, \mathrm{~B}=1\right)$ with downweighting of rare species, Monte-Carlo permutation test was used (reduced model, 499 permutations), forward seletion of environmental variables (both automatic and manual selection) were performed. Analysed environmental variables did not show any collinearity. Their VIF ranged from 1.82 to 5.92. Results of CCA were visualized using Canoco Draw

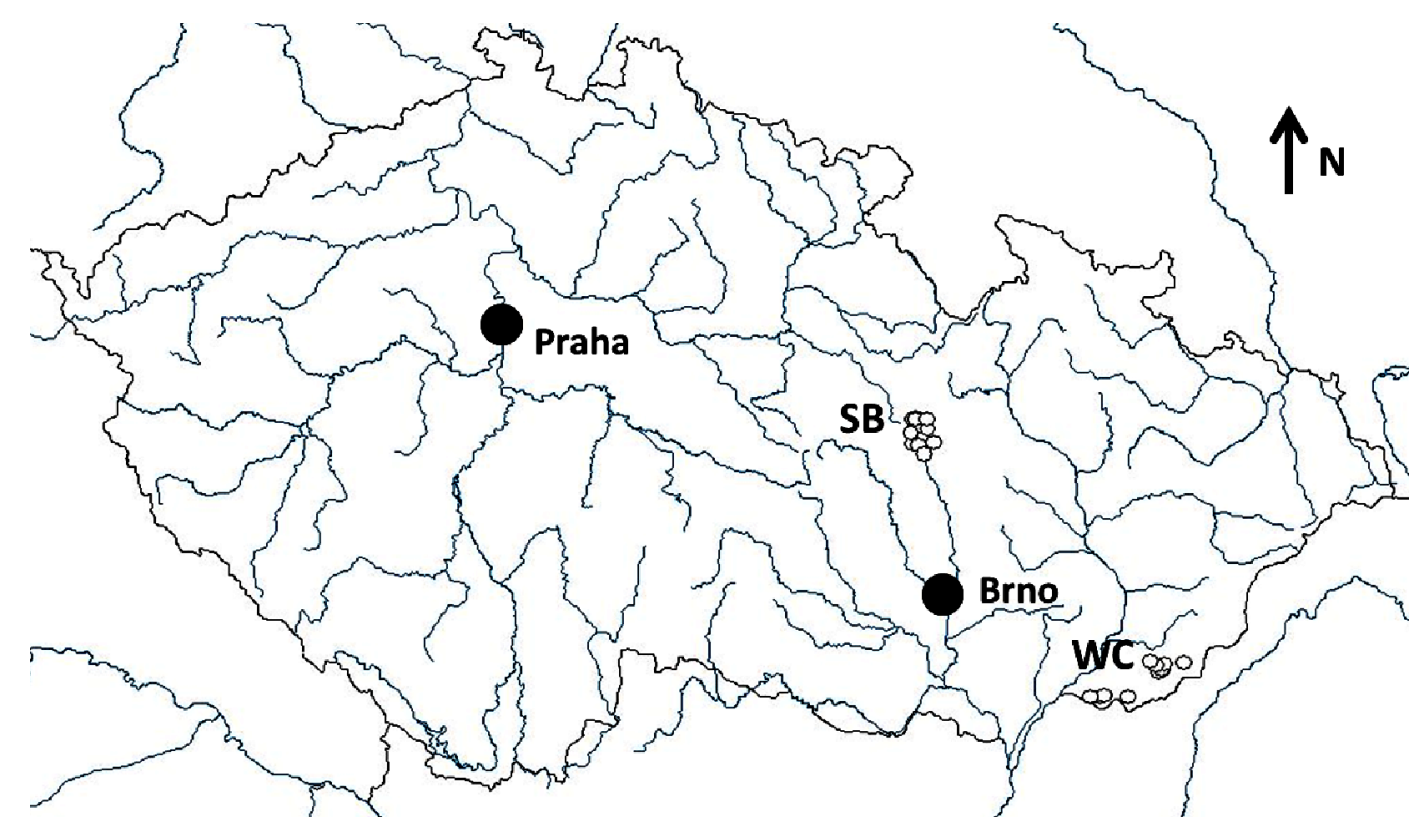

Fig. 1. Sampling sites in White Carpathians (WC) and the Svitava river basin (SB). 
Table. 1. Basic characteristics of investigated localities, (Cond) conductivity $\left(\mu \mathrm{mS} . \mathrm{cm}^{-1}\right)$.

\begin{tabular}{|c|c|c|c|c|c|c|c|}
\hline Area & Sample & $\begin{array}{l}\text { Locality } \\
\text { (Cadaster) }\end{array}$ & GPS coordinates & pH & Cond & $\begin{array}{l}\text { Pond/ } \\
\text { stream }\end{array}$ & Macrophyte \\
\hline WC & LOK1 & $\begin{array}{l}\text { Lučina } \\
\text { (Tvarožná Lhota) }\end{array}$ & $\begin{array}{l}48^{\circ} 51^{\prime} 46.14^{\prime \prime N} \\
17^{\circ} 23^{\prime} 41.04^{\prime \prime} \mathrm{E}\end{array}$ & 8.19 & 505 & $\mathrm{P}$ & Poaceae \\
\hline WC & LOK2 & $\begin{array}{l}\text { Kejda } \\
\text { (Kněždub) }\end{array}$ & $\begin{array}{l}48^{\circ} 52^{\prime} 3.84 " \mathrm{~N} \\
17^{\circ} 24^{\prime} 37.26^{\prime \prime} \mathrm{E}\end{array}$ & 8.13 & 470 & $\mathrm{P}$ & Poaceae \\
\hline $\mathrm{WC}$ & LOK3 & $\begin{array}{l}\text { Radějovka } \\
\text { (Radějov) }\end{array}$ & $\begin{array}{l}48^{\circ} 51^{\prime} 38.16^{\prime \prime} \mathrm{N} \\
17^{\circ} 20^{\prime} 32.70^{\prime \prime} \mathrm{E}\end{array}$ & 8.32 & 546 & S & Poaceae \\
\hline WC & LOK4 & $\begin{array}{l}\text { Hrubý potok } \\
\text { (Javorník) }\end{array}$ & $\begin{array}{l}48^{\circ} 51^{\prime} 49.56^{\prime \prime} \mathrm{N} \\
17^{\circ} 31^{\prime} 54.24^{\prime \prime} \mathrm{E}\end{array}$ & 8.36 & 414 & S & Poaceae \\
\hline WC & LOK5 & $\begin{array}{l}\text { Rasová } \\
\text { (Komňa) }\end{array}$ & $\begin{array}{l}48^{\circ} 58^{\circ} 32.40^{\prime \prime} \mathrm{N} \\
17^{\circ} 48^{\prime} 43.86^{\prime \prime} \mathrm{E}\end{array}$ & 8.24 & 317 & $\mathrm{P}$ & Typha \\
\hline $\mathrm{WC}$ & LOK6 & $\begin{array}{l}\text { Lubná } \\
\text { (Suchá Loz) }\end{array}$ & $\begin{array}{l}48^{\circ} 56^{\prime} 47.88^{\prime \prime} \mathrm{N} \\
17^{\circ} 40^{\prime} 54.60^{\prime \prime} \mathrm{E}\end{array}$ & 7.93 & 451 & $\mathrm{P}$ & Sparganium \\
\hline WC & LOK7 & $\begin{array}{l}\text { Lubná } \\
\text { (Suchá Loz) }\end{array}$ & $\begin{array}{l}48^{\circ} 56^{\prime} 32.16^{\prime \prime} \mathrm{N} \\
17^{\circ} 40^{\prime} 55.80^{\prime \prime} \mathrm{E}\end{array}$ & 8.09 & 523 & S & Poaceae \\
\hline WC & LOK 8 & $\begin{array}{l}\text { Basin on the Hradec- } \\
\text { ký járek } \\
\text { (Suchá Loz) }\end{array}$ & $\begin{array}{l}48^{\circ} 57^{\prime} 1.68^{\prime \prime N} \\
17^{\circ} 42^{\prime} 14.58^{\prime \prime} \mathrm{E}\end{array}$ & 7.79 & 368 & $\mathrm{P}$ & Typha \\
\hline WC & LOK9 & $\begin{array}{l}\text { Hradecký járek } \\
\text { (Suchá Loz) }\end{array}$ & $\begin{array}{l}48^{\circ} 57^{\prime} 8.52 " \mathrm{~N} \\
17^{\circ} 42^{\prime} 5.88^{\prime \prime} \mathrm{E}\end{array}$ & 7.92 & 408 & S & Salix \\
\hline WC & LOK10 & $\begin{array}{l}\text { Nivnička } \\
\text { (Suchá Loz) }\end{array}$ & $\begin{array}{l}48^{\circ} 58^{\prime} 13.20^{\prime \prime} \mathrm{N} \\
17^{\circ} 42^{\prime} 36.30^{\prime \prime} \mathrm{E}\end{array}$ & 8.06 & 539 & S & Poaceae \\
\hline WC & LOK11 & $\begin{array}{l}\text { Basin near Čupák } \\
\text { (Suchá Loz) }\end{array}$ & $\begin{array}{l}48^{\circ} 57^{\prime} 53.52^{\prime \prime} \mathrm{N} \\
17^{\circ} 40^{\prime} 23.344^{\prime \prime} \mathrm{E}\end{array}$ & 8.13 & 430 & $\mathrm{P}$ & Typha \\
\hline WC & LOK12 & $\begin{array}{l}\text { Nivnička } \\
\text { (Nivnice) }\end{array}$ & $\begin{array}{l}48^{\circ} 58^{\prime} 48.00^{\prime \prime} \mathrm{N} \\
17^{\circ} 38^{\prime} 30.84^{\prime \prime} \mathrm{E}\end{array}$ & 8.45 & 547 & S & Poaceae \\
\hline SB & Ra1 & $\begin{array}{l}\text { Radiměřský potok } \\
\text { (Radiměř) }\end{array}$ & $\begin{array}{l}49^{\circ} 41^{\prime} 31.163 " \mathrm{~N} \\
16^{\circ} 27^{\prime} 26.189 " \mathrm{E}\end{array}$ & 6.2 & 175 & S & Poaceae \\
\hline SB & $\mathrm{HnS1}$ & $\begin{array}{l}\text { Dolní hradecký ryb- } \\
\text { níček } \\
\text { (Hradec nad Svita- } \\
\text { vou) }\end{array}$ & $\begin{array}{l}49^{\circ} 41^{\prime} 7.646 " \mathrm{~N} \\
16^{\circ} 28^{\prime} 57.071 " \mathrm{E}\end{array}$ & 7.85 & 743 & $\mathrm{P}$ & Phragmites \\
\hline SB & $\mathrm{HnS} 2$ & $\begin{array}{l}\text { Horní hradecký ryb- } \\
\text { níček } \\
\text { (Hradec nad Sitavou) }\end{array}$ & $\begin{array}{l}49^{\circ} 41^{\prime} 8.094 " \mathrm{~N} \\
16^{\circ} 28^{\prime} 55.294^{\prime \prime} \mathrm{E}\end{array}$ & 4.80 & 534 & $\mathrm{P}$ & Lemna \\
\hline SB & $\mathrm{HnS} 4$ & $\begin{array}{l}\text { Řeka Svitava } \\
\text { (Hradec nad Svita- } \\
\text { vou) }\end{array}$ & $\begin{array}{l}49^{\circ} 41^{\prime} 6.744 " \mathrm{~N} \\
16^{\circ} 28^{\prime} 51.934^{\prime \prime} \mathrm{E}\end{array}$ & 7.00 & 540 & S & Poaceae \\
\hline SB & Sy1 & $\begin{array}{l}\text { Lánský rybník } \\
\text { (Svitavy - Lány) }\end{array}$ & $\begin{array}{l}49^{\circ} 44^{\prime} 35.760 " \mathrm{~N} \\
16^{\circ} 28^{\prime} 8.220^{\prime \prime} \mathrm{E}\end{array}$ & 6.3 & 385 & $\mathrm{P}$ & Phragmites \\
\hline SB & Sy2 & $\begin{array}{l}\text { Svitavský rybník } \\
\text { (Svitavy - Lačnov) }\end{array}$ & $\begin{array}{l}49^{\circ} 45^{\prime} 58.719^{\prime \prime N} \\
16^{\circ} 27^{\prime} 37.682^{\prime \prime} \mathrm{E}\end{array}$ & 6.60 & 401 & $\mathrm{P}$ & Phragmites \\
\hline SB & Sy3 & $\begin{array}{l}\text { Rosnička } \\
\text { (Svitavy - Předměstí) }\end{array}$ & $\begin{array}{l}49^{\circ} 46^{\prime} 15.313^{\prime \prime N} \\
16^{\circ} 27^{\prime} 5.582^{\prime \prime} \mathrm{E}\end{array}$ & 7.98 & 506 & $\mathrm{P}$ & Phragmites \\
\hline SB & Sy6 & $\begin{array}{l}\text { Svitavy } \\
\text { (Svitavy - Lány) }\end{array}$ & $\begin{array}{l}49^{\circ} 44^{\prime} 36.775^{\prime \prime} \mathrm{N} \\
16^{\circ} 28^{\prime} 41.705^{\prime \prime} \mathrm{E}\end{array}$ & 6.90 & 528 & S & Phragmites \\
\hline SB & Sy 8 & $\begin{array}{l}\text { Lačnovský západní } \\
\text { rybník } \\
\text { (Svitavy - Lačnov) }\end{array}$ & $\begin{array}{l}49^{\circ} 46^{\prime} 25.950^{\prime \prime} \mathrm{N} \\
16^{\circ} 28^{\prime} 7.276 " \mathrm{E}\end{array}$ & 5.75 & 293 & $\mathrm{P}$ & Typha \\
\hline SB & Sy12 & $\begin{array}{l}\text { Outlet at Lačnovský } \\
\text { západní rybník } \\
\text { (Svitavy - Lačnov) }\end{array}$ & $\begin{array}{l}49^{\circ} 46^{\prime} 24.584 " \mathrm{~N} \\
16^{\circ} 28^{\prime} 10.762^{\prime \prime} \mathrm{E}\end{array}$ & 6.00 & 251 & $\mathrm{P}$ & Typha \\
\hline
\end{tabular}


Table 1 Cont.

\begin{tabular}{|c|c|c|c|c|c|c|c|}
\hline SB & V1 & $\begin{array}{l}\text { U Rybníčku } \\
\text { (Vendolí) }\end{array}$ & $\begin{array}{l}49^{\circ} 43^{\prime} 33.575^{\prime \prime} \mathrm{N} \\
16^{\circ} 26^{\prime} 39.028^{\prime \prime} \mathrm{E}\end{array}$ & 6.30 & 148 & $\mathrm{P}$ & Lemna \\
\hline SB & Po2 & $\begin{array}{l}\text { Fishpond } \\
\text { (Pohledy) }\end{array}$ & $\begin{array}{l}49^{\circ} 41^{\prime} 46.008^{\prime \prime N} \\
16^{\circ} 33^{\prime} 39.107^{\prime \prime} \mathrm{E}\end{array}$ & 6.30 & 252 & $\mathrm{P}$ & Phragmites \\
\hline SB & KH1 & $\begin{array}{l}\text { Fishpond } \\
\text { (Kamenná horka) }\end{array}$ & $\begin{array}{l}49^{\circ} 44^{\prime} 18.342^{\prime \prime} \mathrm{N} \\
16^{\circ} 31^{\prime} 43.116^{\prime \prime} \mathrm{E}\end{array}$ & 5.80 & 459 & $\mathrm{P}$ & Typha \\
\hline SB & $\mathrm{K} 1$ & $\begin{array}{l}\text { Pool } \\
\text { (Koclířov) }\end{array}$ & $\begin{array}{l}49^{\circ} 46^{\prime} 20.363^{\prime \prime} \mathrm{N} \\
16^{\circ} 31^{\prime} 21.760^{\prime \prime} \mathrm{E}\end{array}$ & 5.88 & 332 & $\mathrm{P}$ & Phragmites \\
\hline SB & $\mathrm{BnS1}$ & $\begin{array}{l}\text { Svitava } \\
\text { (Březová nad Svita- } \\
\text { vou) }\end{array}$ & $\begin{array}{l}49^{\circ} 39^{\prime} 25.761 " \mathrm{~N} \\
16^{\circ} 30^{\prime} 27.857^{\prime \prime} \mathrm{E}\end{array}$ & 6.10 & 552 & $\mathrm{~S}$ & Poaceae \\
\hline SB & $\mathrm{Br} 1$ & $\begin{array}{l}\text { Svitava } \\
\text { (Brněnec) }\end{array}$ & $\begin{array}{l}49^{\circ} 37^{\prime} 23.628^{\prime \prime} \mathrm{N} \\
16^{\circ} 31^{\prime} 26.769^{\prime \prime} \mathrm{E}\end{array}$ & 7.65 & 518 & $\mathrm{~S}$ & Phragmites \\
\hline
\end{tabular}

Table 2. Species complexes occurring in the White Carpathians and the Svitava Basin, their trophic preferences, (nd) no data available.

\begin{tabular}{lll}
\hline Species complex & References & $\begin{array}{l}\text { Trophic state (VAN DAMM et al. } \\
\text { 1994) }\end{array}$ \\
\hline $\begin{array}{l}\text { Achnanthidium minutissimum } \\
\text { Planothidium lanceolatum }\end{array}$ & POTAPOVA \& HAMILTON 2007 & euryvalent \\
Amphora pediculus & VAN DE VIJVER et al. 2013 & eutrophic \\
Cocconeis pediculus & BRUDER 2006, WANG 2014 & eutrophic \\
Cocconeis placentula & JAHN et al. 2007 & eutrophic \\
Encyonema minutum & JAHN et al. 2009 & eutrophic \\
Eunotia bilunaris & & nd \\
Ulnaria ulna & VANORMELINGER et al. 2013 & euryvalent \\
Fragilaria capucina & WILLIAMS 2011 & euryvalent \\
Staurosirella pinnata & KAHLERT et al. 2009 & euryvalent \\
Gomphonema parvulum & MORALES et al. 2013 & euryvalent \\
Navicula cryptocephala & ABARCA et al. 2014 & eutrophic \\
Nitzschia palea & POULÍČKOVÁ et al. 2010 & euryvalent \\
Nitzschia paleacea & KAHLERT et al. 2009; TROBAJO et al. 2009 & hypertrophic \\
Sellaphora pupula & & eutrophic \\
& MANN et al. 2004, 2008; VANORMELINGEN et & mesotrophic \\
\hline
\end{tabular}

4.0. Shannon diversity indexes of diatoms were computed (then sorted according to: host plants, sampling site, streaming/stagnant water, geographical location of sampling sites). Variation of Shannon diversity indexes among sampling sites and streaming/stagnant water was analysed with OneWay ANOVA. Because of unequal number of host plants sampled, variation of Shannon diversity index was analysed with non- parametric Kruskal-Wallis multiple comparison test (NCSS statistical package, HinTZE 2007). With respect to different plant habitus and physiology, difference in Shannon's diversity was analysed for Lemna minor versus group of other submerged vascular plants. Response of diatoms to the best fitting environmental variables was anlysed using $\mathrm{T}-$ value statistics (CanocoDraw 4.0).

\section{Results}

A total of 131 diatom species was found during the study. Species richness ranged from 1 to 34 species per sample. Species richness was higher in the West Carpathians (19-34) than in the Svitava river basin (1-15 per sample). The highest number of diatom species was found on Poaceae and Typha. The dominant diatom species was Achnanthidium minutissimum agg. creating up to $88 \%$ of the community. The most frequently occuring species were Gomphonema parvulum agg. with representation $1-48 \%$ and Cocconeis placentula with representation $1-100 \%$. Frequent species for both regions were also Nitzschia palea and Ulnaria ulna. Planktonic diatoms (Cyclotella, Aulacoseira and Asterionella) frequently occurred in ponds. 
Surprisingly species complexes (Table 2) represent the majority of epiphytic assemblages (lotic/lentic: $67,1 \%$ and 66,5\% respectively; substrates: Poaceae 63\%, Typha $60 \%$, Sparganium 79,2\%, Salix 74.9\%, Phragmites 75\%, Callitriche 89,8\%, Lemna 25\%). However, differences in percentage of species complexes among sampled host plants were not significant $(\mathrm{F}=1.91, \mathrm{P}=$ 0.1341 ). There was no close correlation between Shannon's diversity and percentage of species complexes among sampled host plants as well (Pearson correlation coefficient: $r=0.4113$ ) by other words: the high representation of complexes does not automatically lead to reduction of overall diversity of the sample.

Canonical correspondence analysis spread sampling points through the ordination spacewith respect to their geographical position (West Carpathians and Svitava river basin) and ecological nature (stream and pond). Sampling sites in the Western Carpathians, both ponds and streams, form more coherent clusters than in the Svitava river basin (Fig 3). Significant differences in Shannon's diversity index were found between sampling sites in the Svitava river basin and Western Carpathians (Fig. 6, F = 5.88, p = 0.0204). Sampling sites located in the Western Carpathians (Fig. 3, squares) possess statistically higher Shannon diversity $(1.95 \pm 0.55)$ than those in the Svitava river basin $(1.43 \pm 0.68)$. Similarly, statistically significant differences were found between sampling sites from streaming $(2.08 \pm 0.47)$ and stagnant water bodies $(1.33 \pm 0.63$; Fig. $5, \mathrm{~F}=13.94, \mathrm{p}=0.0006)$.

Species data explain $11.8 \%$ of variability on the first and $19.8 \%$ on the second ordination axis $(\mathrm{p}=0.002, \mathrm{~F}$ $=3.792$ ). Diatom ordination is significantly influenced by $\mathrm{pH}(\mathrm{F}=3.84, \mathrm{p}=0.002)$, water hydrodynamism (streaming/stagnant, $\mathrm{F}=2.59, \mathrm{p}=0.002$ ) and Lemna minor as a host plant $(\mathrm{F}=1.97, \mathrm{p}=0.018$, for details see Table 3, Fig. 4). Species such as Amphora pediculus, Cocconeis pediculus, Cymbella excisiformis, Encyonopsis cesatii, Encyonopsis microcephala, Eunotia arcus, Gomphonema pumilum, Nitzchia palaeformis or Nitzchia sinuata prefer significantly higher $\mathrm{pH}$ than Mayamaea atomus, Planothidium ellipticum, Planothidium lanceolatum or Nitzchia palea, which prefer lower $\mathrm{pH}$ value within investigated scale (4.80 $8.45)$. Species preferring stagnant water bodies include Fragilaria brevistriata, Encyonopsis microcephala, Eunotia arcus and Denticula tenuis. On the other hand, streaming water prefer Cocconeis pediculus, Gomphonema angustatum and Navicula tripunctata. Diatom assemblages among sampled host plants possess almost the same diversity. Lemna minor showed the lowest variability of Shannon diversity $(1.37 \pm 0.17)$ in contrast to other host plants (Phrag $=1.56 \pm 0.72$, Poac $=1.82 \pm 0.53$, Salix $=1.54 \pm 1.38$, Typha $=1.50 \pm 0.59$ ). However, Shannon's diversity did not show any statistically significant differences among host plants (Table 4). Similarly, difference in Shannon's diversity

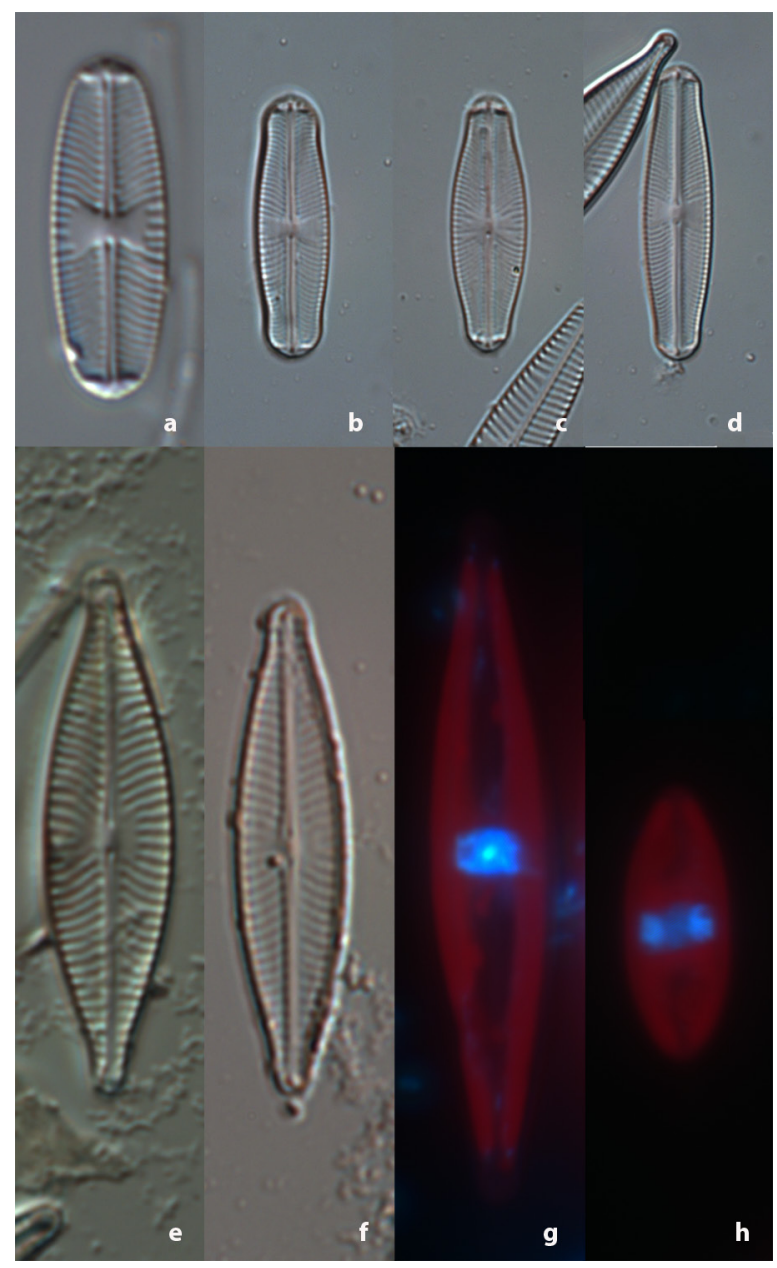

Fig. 2. Examples of species complexes in the Czech Republic: (a-d) Sellaphora pupula sensu lato differ in frustule morphology, (e-h) Navicula cryptocephala sensu lato differ in interphase nuclei structure.

between Lemna minor and other vascular submerged plants was not significant as well $(\mathrm{z}=0.874, \alpha=0.05)$. Diatoms showed low specificity to host plants except of Lemna minor $(\mathrm{F}=1.97, \mathrm{p}=0.018)$. Species such as Fragilaria brevistriata, Staurosirella pinnata or Nitzschia palaeformis avoid Lemna minor as a host plant. Surprisingly, Lemnicola hungarica as a diatom typical for Lemna minor, inhabited also Phragmites australis in the Svitava river basin.

\section{Discussion}

Small streams and shallow ponds represent ecosystems sensitive to environmental changes. It can be demonstrated by much higher nutrient variation in shallow than deep waters (JEPPESEN et al. 2000). In comparison with physicochemical variables, attached diatoms seem to be more integrative indicators with fast response to environmental changes (BLANCO et al. 2004; HÁsek et al. 2014). Diatoms are able to inhabit all available substrates, which are mostly represented by 


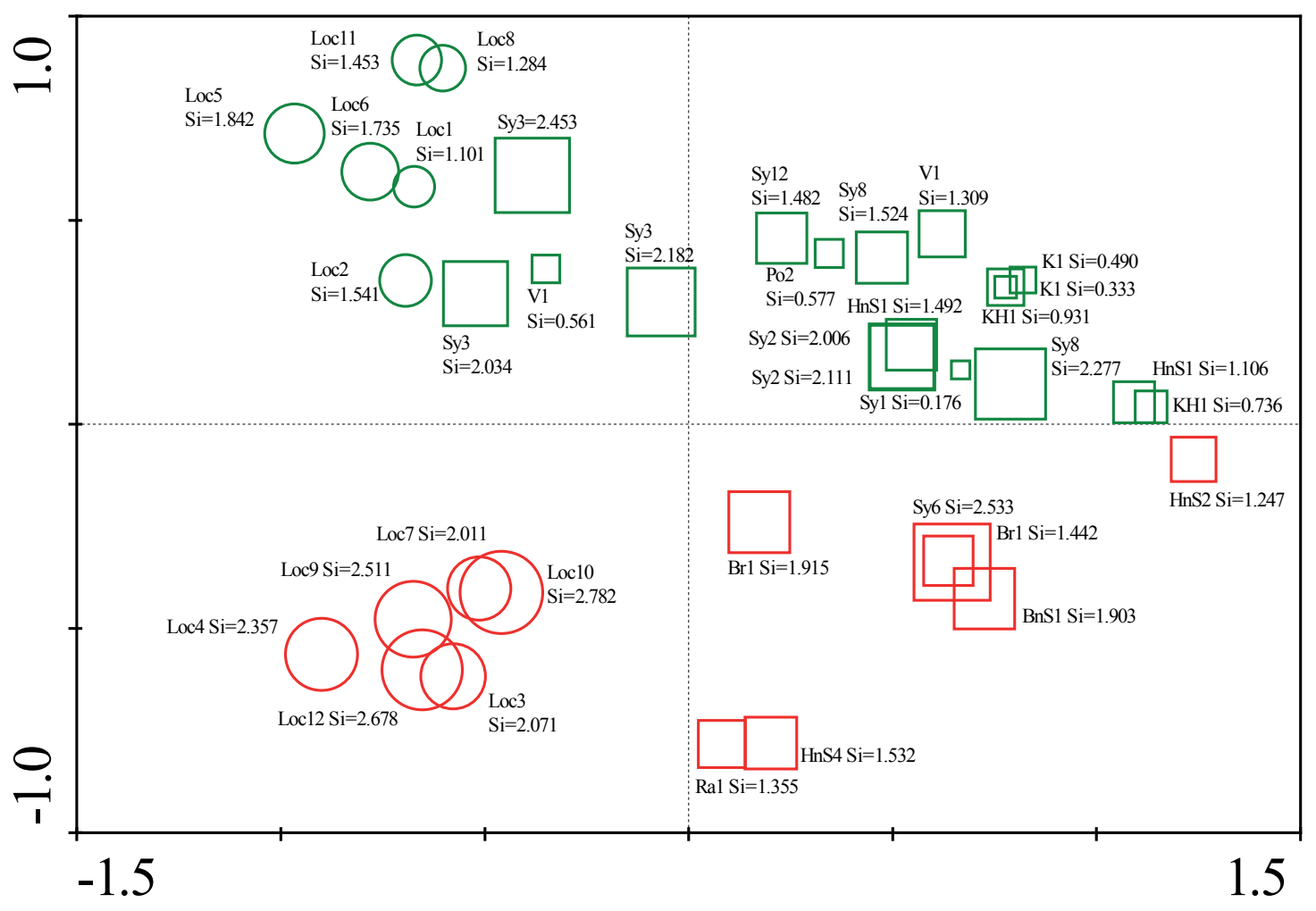

Fig. 3 CCA ordination diagram: investigated sites, (circles) Western Carpathians, (squares) Svitava river basin, (green) ponds, (red) streams; symbol size corresponds to value of Shannon's diversity index (Si).

Fig. 4. CCA ordination biplot diagram: species vs. environmental variables: (Achmin) Achnanthidium minutissimum (KüTZING) CZARNECKI, (Adlsp) Adlafia sp, (Ampel) Amphipleura pellucida (Kützing) Kützing, (Ampova) Amphora ovalis (KütZing) KüTZing, (Ampped) Amphora pediculus (KütZing) Grunow ex A. Schmidt, (Ampsp) Amphora sp, (Ampsp2) Amphora sp. 2, (Ampsp3) Amphora sp. 3, (Astfor) Asterionella formosa Hassall, (Aulgra) Aulacoseira granulata (Ehrenberg) Simonsen, (Braneo) Brachysira neoexilis Lange-Bertalot, (Cocped) Cocconeis pediculus Ehrenberg, (Cocpla) Cocconeis placentula Ehrenberg, (Cabud) Craticula buderi (Hustedt) Lange-Bertalot, (Cyccom) Handmannia comta (Ehrenberg) Kociolek et Khursevich, (Ccdis) Cyclotella distinguenda Hustedt, (Cycmen) Cyclotella meneghiniana Kützing, (Cycsp) Cyclotella sp., (Cymeli) Cymatopleura elliptica (Brébisson) W. Sмiтн, (Cymcym) Cymbella cymbiformis C. Agardh, (Cymexc) Cymbella excisiformis Krammer, (Cymlan) Cymbella lanceolata (C.Agardh) Kirchner, (Cymamp) Cymbopleura amphicephala (NÄGeli) Krammer, (Deldel) Delicata delicatula (Kützing) Krammer, (Denten) Denticula tenuis Kützing, (Diaten) Diatoma tenuis agg., (Diavul) Diatoma vulgaris Bory de Saint-Vincent, (Dippet) Diploneis petersenii Hustedt, (Dipsep) Diploneis separanda Lange-Bertalot, (Enccae) Encyonema caespitosum KüTZIng, (Encmin) Encyonema minutum agg., (Encces) Encyonopsis cesatii (RABenhorst) Krammer, (Encmic) Encyonopsis microcephala agg., (Eucfle) Eucocconeis flexella (KüTZING) MeIster, (Euclae) Eucocconeis laevis (Østrup) Lange-Bertalot, (Eunarc) Eunotia arcus Ehrenserg, (Fallen) Fallacia lenzii (Hustedt) Lange-Bertalot, (Falpyg) Fallacia pygmaea (Kützing) A.J. Stickle et D.G. Mann, (Falsp1) Fallacia sp., (Falsp3) Fallacia sp. 3, (Falsub) Fallacia subhamulata (Grunow) D.G. Mann, (Fissp) Fistulifera sp., (Fraacu) Fragilaria acus (KütZing) Lange-Bertalot, (Frabe) Fragilaria brevistriata Grunow, (Fracap) Fragilaria capucina Desmazières, (Franan) Fragilaria nanana Lange-Bertalot, (Frapar) Fragilaria parasitica (W. Sмiтh) Grunow var. parasitica, (Frasp) Fragilaria sp., (Frapin) Staurosirella pinnata (EhrenBerg) D. M. Williams et Round, (Frasp2) Fragilaria sp. 2, (Frasp3) Fragilaria sp., (Gomacu) Gomphonema acuminatum Ehrenberg, (Gomang) Gomphonema angustatum (KütZING) RABEnhorst, (Gomcor) Gomphonema coronatum (EhrenBerG), (Gomoli) Gomphonema olivaceum (Hornemann) BréBIsson, (Gompar) Gomphonema parvulum (KÜTZING) KÜTZING, (Gompum) Gomphonema pumilum (Grunow) E. Reichardt et Lange-Bertalot, (Gomtru) Gomphonema truncatum Ehrenberg, (Gyracu) Gyrosigma acuminatum (Kützing) RABenhorst, (Hipcap) Hippodonta capitata (Ehrenberg) Lange-Bertalot, Metzeltin et Witkowski, (Lemhun) Lemnicola hungarica (Grunow) F.E.Round et P.W.Basson, (Lutgoe) Luticola goeppertiana (Bleisch) D.G. Mann, (Mayato) Mayamaea atomus (Kützing) Lange-Bertalot, (Melvar) Melosira varians C. Agardh, (Mercir) Meridion circulare (Greville) C.AgardH, (Micsp) Microcostatus sp., (Navant) Navicula antonii Lange-Bertalot, (Navci) Navicula cryptotenelloides Lange-Bertalot, (Navgre) Navicula gregaria Donkin, (Navmen) Navicula menisculus Schumann, (Navnot) Navicula notha WAllace, (Navrad) Navicula radiosa Kützing, (Navsp2) Navicula sp. 2, (Navtri) Navicula tripunctata (O.F. Müller) Bory de Saint-Vincent, (Navtriv) Navicula trivialis Lange-Bertalot, (Navven) Navicula veneta Kützing, (Nitaci) Nitzschia acicularis (KützING) W.Smith, (Nitamp) Nitzschia amphibia Grunow, (Nitang) Tryblionella angustata W. Smith, (Nitcon) Nitzschia constricta (KütZing) Ralfs, (Nitdis) Nitzschia dissipata (KütZing) Rabenhorst, (Nitfon) Nitzschia fonticola (Grunow) Grunow, (Nithun) Tryblionella hungarica (Grunow) Frengueldi, (Nitfru) Nitzschia inconspicua Grunow, (Nitlin) Nitzschia linearis W. Smith, (Nitplf) Nitzschia palaeformis Hustedt, (Nitdeb) Nitzschia palea var. debilis (Kützing) Grunow, (Nitpal) Nitzschia palea (Kützing) W.Smith, (Nitplc) Nitzschia paleacea Grunow, (Nitsin) Grunowia sinuata (Thwaites) Rabenhorst, (Nitver) Nitzschia vermicularis (Kützing) Hantzsch, (Pinnob) Pinnularia nobilis (Ehrenberg) Ehrenberg, (Plaell) Planothidium ellipticum (Cleve) Round et Bukhtiyarova, (Plalan) Planothidium lanceolatum (BréBisson ex Kützing) Bukhtiyarova, (Reisin) Reimeria sinuata (Gregory) Kociolek et Stoermer, (Rhoabb) Rhoicosphenia abbreviata (C.Agardh) Lange-Bertalot, (Rhogib) Rhopalodia gibba (Ehrengerg) Otto Müller, (Selpup) Sellaphora pupula (KütZing) MereschKowsky, (Stagra) Stauroneis gracilis EhrenBerg, (Surbre) Surirella brebissonii var. kuetzingii KRAMmer et LANGE-BERTAlot, (Surova) Surirella ovalis BRÉBISSON, (Ulnuln) Ulnaria ulna (Nitzsch) P.Compère. Variables: (Water) water streaming 0/1, (Cond) conductivity, pH, (Shadow) percentage of shade 0\%/50\%/100\%; substrates: Phragmites, Poaceae, Typha, Lemna, Salix, Callitriche, Sparganium. 


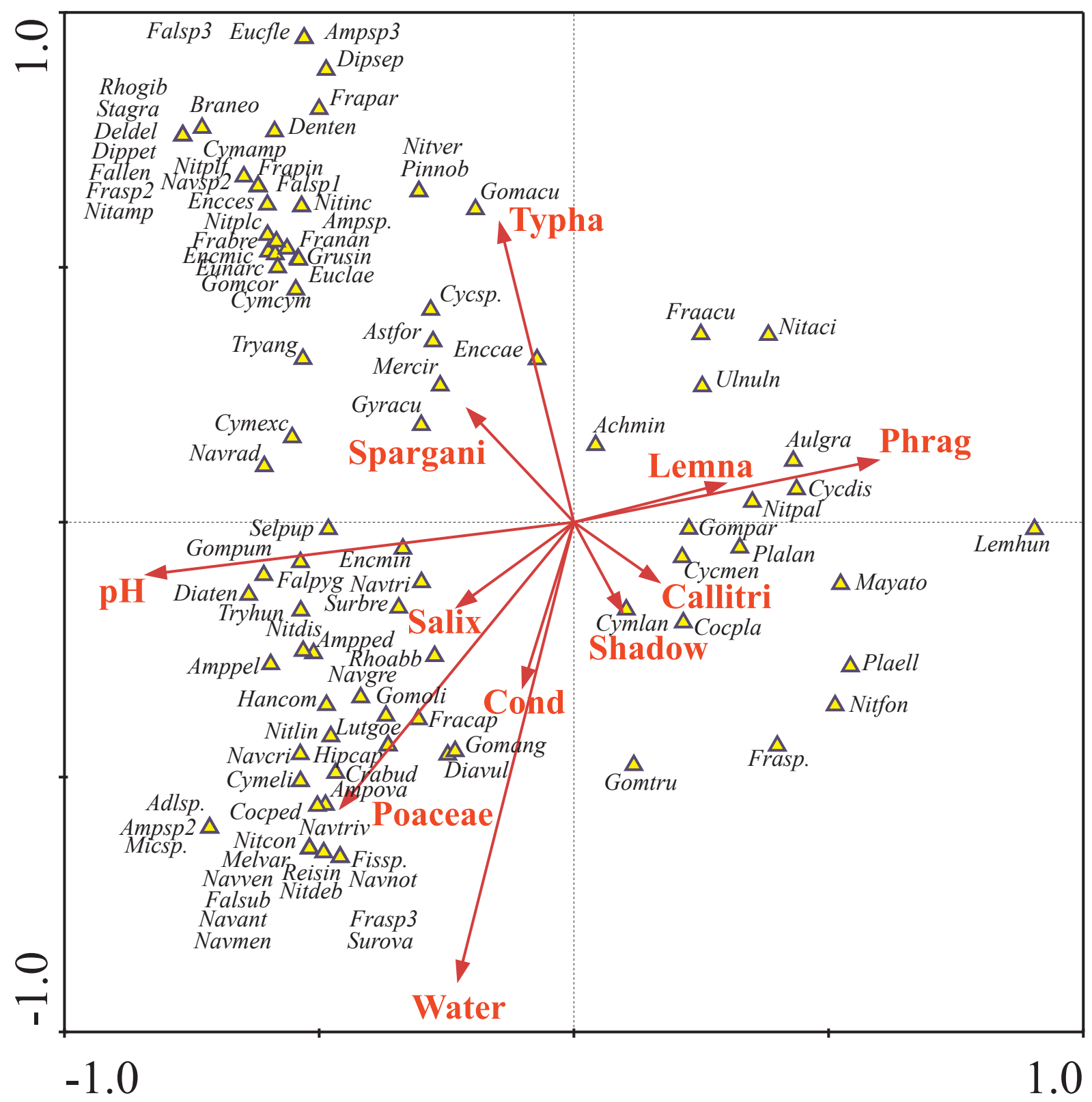

stones and sediments in streams and by macrophytes and sediments in shallow lakes and ponds. Homogeneity of periphytic communities and their composition are more related to chemical characteristics of the surrounding environment than to the substrate type, particularly in eutrophic systems (EMINSON \& Moss 1980; Cattaneo et al. 1998; Kitner \& PoulíčKová 2003; PoulíčKová et al. 2004; Cejudo-Figueiras et al. 2010). However, substrate specificity has been described in some oligotrophic waters (EMINSON \& Moss 1980; Blindow 1987; Buczkó 2006; CAntonaTI 1998; PoulíčKovÁ et al. 2004). Our results did not confirm substrate specificity, except of specific assemblage growing on Lemna sp., similar to more complex study already published (BUCZKó 2007). In contrast to other periphytic assemblages, epiphytic assemblages include lower number of planktonic diatom taxa and suspended particules (Kelly et al. 1998, PoulíčKová et al. 2004, 2008, 2014). Planktonic diatoms were not frequent and were represented by Aulacoseira granulata (SB), Asterionella formosa and Cyclotella sp. (WC). In general, the relationship between epiphyton and water chemistry has been demonstrated many times (Ács et al. 1991, 1994; Kitner \& PoulíčKovÁ 2003; Blanco et al. 2004; PoulíčKovÁ et al. 2004; HÁJKovÁ et al. 2011) and submerged macrophytes have been recommended for routine monitoring (Kelly et al. 1998; BLANCO et al. 2004). We found significant relationship to selected environmental variables (water streaming, $\mathrm{pH}$ ) in congruence with other studies (PotAPOVA \& CHARLES 2003; Kitner \& PoulíčKová 2003; KovÁcs et al. 2006; FrÁNKOvÁ et al. 2009; YANG \& FLOWER 2012).

However, the results of this method of water quality status assessment are strongly influenced by following 


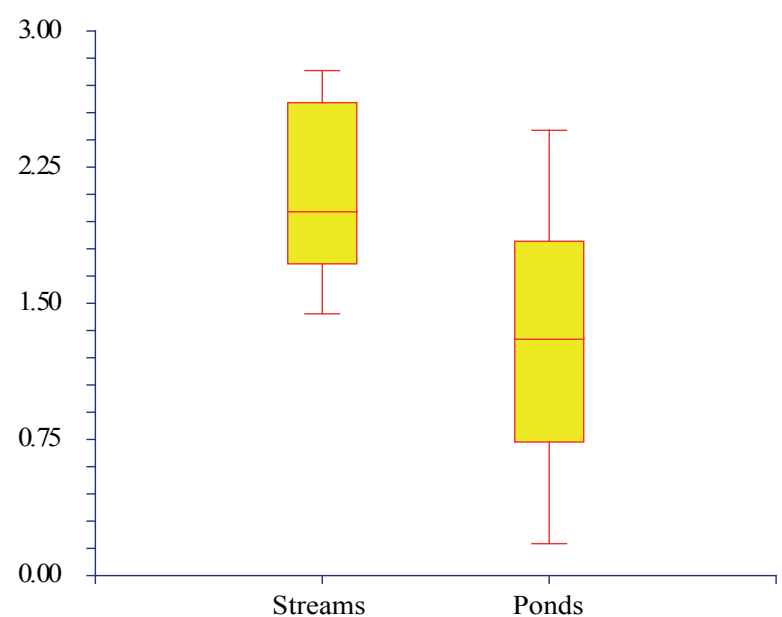

Fig. 5. Box plot of diatom Shannon diversity: comparison of habitat (streams, ponds; $\mathrm{F}=13.94, \mathrm{p}=0.0006$ ).

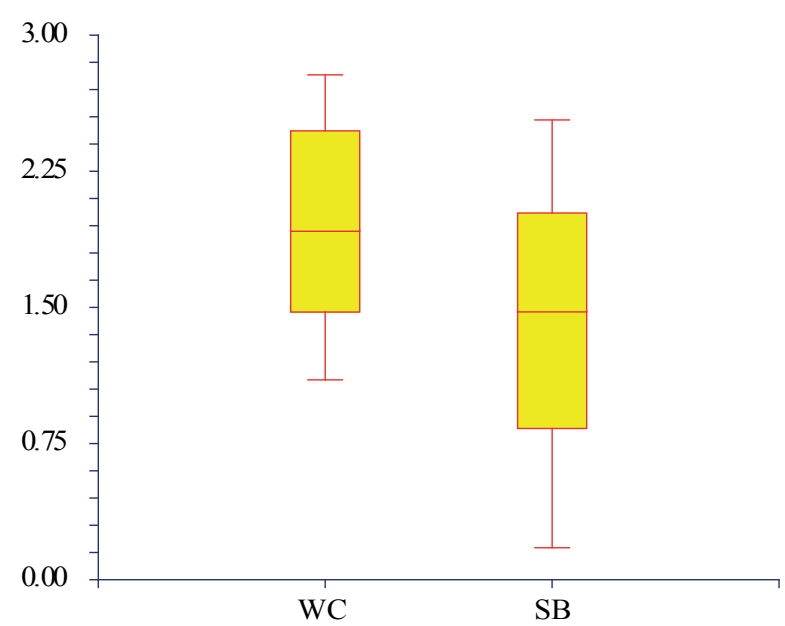

Fig. 6. Box plot of diatom Shannon diversity: comparison of sampling sites (SB) Svitava river basin, (WC) Western Carpathians $(\mathrm{F}=5.88, \mathrm{p}=0.0204)$.

two factors: 1) trophic indices are working in ecoregions where they were intercalibrated (PRYGIEL et al. 2002; PoulíčKOvÁ et al. 2004; KovÁc et al. 2006) and 2) some traditional ,euryvalent and cosmopolitan species" represent species complexes consisting of few or many ecologically differentiated biological species (so called cryptic species), whose distinguishing in the LM is difficult or even impossible (MANN et al. 2008; KAHLERT et al. 2009; PouLíčKovÁ et al. 2010).

Although many studies noticed that attached diatoms in wide spectrum of ecological conditions are dominated particularly by Achnanthidium minutissimum (Kützing) Czarnecki 1994 (Blanco et al. 2004, Cejudo-Figueiras et al. 2010), the assessment what is the proportion of species complexes within attached diatom assemblages has not been specified yet. SigEE (2005) summarized dominant diatom species along a river course with increasing nutrient pollution. First zone (clean water) is characterized by small-celled species directly attached to stone surface (Eunotia exigua, Achnanthes microcephala). Zones 2 and 3 are dominated by Fragilaria capucina, Achnanthidium minutissimum, Encyonema minutum and Cocconeis placentula. Eutrophic zone 4 is characterized by Gomphonema parvulum and highly eutrophic zone 5 by Nitzschia palea (SigEe 2005). In fact the majority of these ,indicatory dominants“ represent species complexes (Table 2; KWANDrAns et al. 1998; KAHLERT et al. 2009). If species complexes as a whole are not ecologically differentiated and create the majority of assemblage composition, it means that in this field is a great potential for trophic indices improvement. The ongoing progress with identification of cryptic diversity is in motion with implementation of molecular methods. Following six examples demonstrates the importance of species complexes investigation.

\section{Sellaphora pupula agg.}

An extreme example of species complexes seems to be Sellaphora pupula agg. (Fig. 2; MANn et al. 2008) with almost 50 morphospecies (demes), some of them already confirmed using molecular methods (EvANs et al. 2007, 2009; Wetzel et al. 2015). This diatom is typical for epipelic rather than epiphytic assemblages, with high representation in British lakes. It creates up to $40 \%$ of epipelic assemblages in lakes/ponds of Great Britain, while its representation in Czech and Hungarian ponds does not exceed 3\% (PoulíčKovÁ et al. 2008, ŠPAČKOVÁ et al. 2009). Although their identification is difficult particularly in the LM, some of them seem to be ecologically differentiated (PoulíčKOvÁ et al. 2008). Many lakes contain several different morphospecies, the greatest numbers of coexisting demes occurred in eutrophic Blackford Pond, Great Britain (PoulíčKová et al. 2008). Five morphospecies inhabiting Czech pond Bezedník (temperate zone) showed seasonal dynamics with significant correlation of their occurrence with temperature (ŠPAČKOvÁ et al. 2009).

\section{Achnanthidium minutissimum agg.}

Although molecular methods have not been used in this case yet, the opinion that previously described varieties within $A$. minutissimum can represent ecologically differenctiated species seems to be evident (PotAPova \& Hamilton 2007). Moreover this species complex has been recorded as the most frequent dominant of epilithic and epiphytic assemblages in both lotic and lentic freshwaters (Ponader \& Potapova 2007; Potapova \& Hamilton 2007). Morphometric study (Potapova \& HamiLton 2007) revealed 6 morphological groups, however authors were not able to draw clear boundaries between them. These morphospecies differred significantly in their ecology and could serve as indicators of water quality (PotAPOVA \& HAMILTON 2007). However, an analysis of the results of 25 diatomists participating in intercalibration exercise showed, that even experienced diatomists have problem to recog- 
Table 3. CCA forward selection of environmental variables: influence of environmental variables on species distribution in the Svitava river basin and the White Carpathian Mts [(Water) streaming/stagnant water, (Cond) conductivity, (Lemna) Lemna minor, (Spargani) Sparganium sp., (Shadow) shadow/half-shadow/light, (Typha) Typha spp., (Phrag) Phragmites australis, (Callitri) Callitriche sp.].

\begin{tabular}{llllllll}
\hline \multicolumn{2}{l}{ Conditional Effects } & & \multicolumn{5}{c}{ Marginal Effects } \\
Variable & Var.N & LambdaA & $\mathbf{p}$ & $\mathbf{F}$ & Lambda1 & $\mathbf{p}$ & $\mathbf{F}$ \\
\hline pH & 1 & 0.32 & $\mathbf{0 . 0 0 2}$ & $\mathbf{3 . 8 4 * *}$ & 0.32 & $\mathbf{0 . 0 0 2}$ & $\mathbf{3 . 8 4 * *}$ \\
Water & 4 & 0.21 & $\mathbf{0 . 0 0 2}$ & $\mathbf{2 . 5 9 * *}$ & 0.22 & $\mathbf{0 . 0 0 4}$ & $\mathbf{2 . 5 0 * *}$ \\
Cond & 2 & 0.16 & $\mathbf{0 . 0 1 0}$ & $\mathbf{2 . 1 0 * *}$ & 0.13 & 0.090 & 1.41 \\
Lemna & 8 & 0.15 & $\mathbf{0 . 0 1 8}$ & $\mathbf{1 . 9 7 * *}$ & 0.17 & $\mathbf{0 . 0 2 4}$ & $\mathbf{1 . 8 8 * *}$ \\
Spargani & 11 & 0.10 & 0.254 & 1.27 & 0.11 & 0.358 & 1.21 \\
Shadow & 3 & 0.08 & 0.278 & 1.14 & 0.09 & 0.406 & 1.1 \\
Typha & 7 & 0.08 & 0.314 & 1.7 & 0.13 & 0.078 & 1.51 \\
Poaceae & 6 & 0.08 & 0.434 & 1.1 & 0.18 & $\mathbf{0 . 0 0 4}$ & $\mathbf{2 . 3 * *}$ \\
Salix & 9 & 0.08 & 0.452 & 1.00 & 0.09 & 0.464 & 0.99 \\
Phrag & 5 & 0.03 & 0.920 & 0.41 & 0.20 & $\mathbf{0 . 0 0 2}$ & $\mathbf{2 . 3 3 * *}$ \\
Callitri & 10 & & & & 0.05 & 0.796 & 0.57 \\
** statistically significant & & & & &
\end{tabular}

Table 4. Kruskal-Wallis Multiple-Comparison Z-Value Test (Dunn's Test), differences of Shannon's diversity index among host plants [(Lemna) Lemna minor, (Poac) Poaceae, (Phrag) Phragmites australis, (Salix) Salix spp., (Typha) Typha spp.].

\begin{tabular}{l|rrrrr}
\hline & \multicolumn{1}{|l}{ Lemna } & Poac & Phrag & Salix & \multicolumn{2}{c}{ Typha } \\
\hline Lemna & 0.0000 & 1.1867 & 0.8036 & 0.5970 & 0.4306 \\
Poac & 1.1867 & 0.0000 & 0.6897 & 0.4315 & 1.1457 \\
Phrag & 0.8036 & 0.6897 & 0.0000 & 0.0269 & 0.5636 \\
Salix & 0.5970 & 0.4315 & 0.0269 & 0.0000 & 0.3141 \\
Typha & 0.4306 & 1.1457 & 0.5636 & 0.3141 & 0.0000 \\
\hline
\end{tabular}

Regular Test: Medians significantly different if $z$-value $>1.9600$

Bonferroni Test: Medians significantly different if $z$-value $>2.8070$

nize varieties/morphospecies of $A$. minutissimum in the LM (KAHLERT et al. 2009), due to small size close to the LM resolution limits (length $5-25 \mu \mathrm{m}$, width $2.5-4 \mu$ and dense striation $26-30 / 10 \mu \mathrm{m}$; HofmANn et al. 2013). Improvement in bioassessments in this case strongly depends on application of molecular methods.

\section{Gomphonema parvulum agg.}

The name $G$. parvulum represents a diatom species which is relatively small in size (length $10-36 \mu \mathrm{m}$, width 5-8 $\mu \mathrm{m}$ ) and has cosmopolitan distribution (HoFMANN et al. 2013). In fact it has been used as a collective name for a species complex for two centuries. Morphologically highly variable diatom occurrs in wide range of water qualities (PATRICK \& REIMER 1975; Hudstedt 1985; Krammer \& Lange-Bertalot 1997). Molecular as well as morphological data obtained during the recent studies (KERMAREC et al. 2013; ABARCA et al. 2014) resulted in separation of at least four taxa based on their biogeography.

\section{Eunotia bilunaris agg.}

E. bilunaris sensu lato is a good candidate for studies on semicryptic species diversity in diatoms. It is a cosmopolitan and common epiphytic diatom in oligotrophic, mainly acidic freshwater bodies (KRAMMER \& LANGE-Bertalot 1991; VANORMELINGEn et al. 2008). Based on its phenotypic plasticity, a number of species have been described (LANGE-BERTALOT et al. 2011). Moreover morphological, molecular and reproductive data suggest the existence of several reproductivelly isolated species (VARNORMELINGEN et al. 2008).

\section{Navicula cryptocephala agg.}

$N$. cryptocephala is a common benthic diatom of moderate size (20-40 um long, 5-7 $\mu \mathrm{m}$ wide; LANGE-BERTALOT 2001).

In contrast to species complexes with broad morphological variation, $N$. cryptocephala represents a complex with almost identical valve morphology. However, it has been found to be polymorphic with respect to interphase nuclear structure (Fig. 2; GEITLER 1951, 1952a,b, 1958; PoulíčKovÁ et al. 2010). Phylo- 
genetic analyses of 52 strains confirmed the existence of two genetically distinct lineages within $N$. cryptocephala that coexist sympatrically and are widely distributed, occurring in European and Australian ponds (PoulíčKovÁ et al. 2010).

\section{Nitzschia palea agg.}

$N$. palea is believed to be a widely distributed diatom in lotic and lentic freshwater habitats (FINLAY et al. 2002; Potapova \& Charles 2007). In general, the genus Nitzschia is difficult for identification and discrimination between members, particularly in the section Lanceolatae Grunow (HustedT 1930). Moreover identification is complicated by morphological variability during the life cycle and phenotypic plasticity due to environmental conditions. On the base of their results (morphological, genetic and mating diversity) ТRовало et al. 2009 concluded that $N$. palea is not a simple, homogeneous taxon and that this complex will probably have to be split into three or more species. At least two of them appear to be geographically widespread. Ecological preferences and potential indicatory value need to be further investigated (TROBAJo et al. 2009).

In conclusion, species complexes are important, because of their common occurrence, frequent dominance and difficulties with their distinguishing.

\section{ACKNOWLEDGEMENT}

This study was supported as a long-term research development project no. RVO 67985939 and IGA PrF-2015-001.

\section{REFERENCES}

Abarca, N.; Jahn, R.; Zimmermann, J. \& EnKe, N. (2014): Does the cosmopolitan diatom Gomphonema parvulum (Kützing) Kützing have a biogeography? - PLoS One 9: 1

Ács, É.; Buczkó, K. \& Lakatos, G. (1991): A Velencei-Tó és a Ferto nádbevonatának összehasonlító algológiai elóvizsgálata (Comparative algological study of the reed-periphyton in lake Velencei and lake Ferto). Bot. Közlem. 78: 95-111.

Ács, É.; Buczkó, K. \& LaKatos, G. (1994): Changes in the mosaic-like water surfaces of the Lake Velence as reflected by reed periphyton studies. - Studia Bot. Hung. 25: 5-19.

Blanco, S.; Cejudo-Figueiras, C.; Álvarez-Blanco, I.; Van Donk, E.; Gross, E.M.; Hansson, L.-A.; Irvine, K.; Jeppensen, E.; Kairesalo, T.; Moss, B.; Nõges, T. \& BÉCAREs, E. (2014): Epiphytic diatoms along environmental gradients in western european shallow lakes. - Clean soil air water 42(3): 229-235.

Blanco, S.; Ector, L. \& BéCAREs, E. (2004): Epiphytic diatoms as water quality indicators in spanish shallow lakes. - Vie Milieu 54: 71-79.

BLINDOw, I. (1987): The composition and density of epiphyton on several species of submerged macrophytes. The neutral hypothesis tested. - Aquat. Bot. 29: 157168.
BRUDER, K. (2006): Taxonomic revision of diatoms belonging to the family Naviculaceae based on morphological and molecular data. - 141 pp., Dissertation zur Erlangung des Akademischen Grades eines der Naturwissenschaften. Universität Bremen.

Buczkó, K. (2006): Bryophitic diatoms from Hungary. - Eighteenth International Diatom Symposium Miedzyzdroje, Poland, A. Witkovski [ed.] - Biopress limited, Bristol: 1-15.

BuczKó, K. (2007): The occurrence of the epiphytic diatom Lemnicola hungarica on different European Lemnaceae species. - Fottea 7(1): 77-84.

Cantonati, M. (1998): Diatom communities of springs in the southern Alps. - Diatom Research 13: 201-220.

Cattaneo, A.; Gaetano, G.; Gentinetta, S. \& Romo, S. (1998): Epiphytic algae and macroinvertebrates on submerged and floating-leaved macrophytes in an italian lake. - Freshwater Biol. 39: 725-740.

Cejudo-Figueras, C.; Álvarez-Blanco, I.; Bécares, E. \& Blanco, S. (2010): Epiphytic diatoms and water quality in shallow lakes: the neutral substrate hypothesis revisited. - Mar. Freshwater. Res. 61: 1457-1467.

Eminson, D. \& Moss, B. (1980): The composition and ecology of periphyton communities in freshwaters. 1: The influence of the host type and external environment on community composition. - Br. Phycol. J. 15: 429-446.

Evans, K.M.; Chepurnov, V.A.; Sluiman, H.J.; Thomas, S.J.; SPEARs, B.M. \& ManN, D.G. (2009): Highly differentiated populations of the freshwater diatom Sellaphora capitata suggest limited dispersaland opportunities for allopatric. - Protist 160: 386-396.

Evans, K.M.; WortLey, A.H. \& ManN, D.G. (2007): An assessment of potential diatom "barcode" genes (cox1, rbcL, 18S and ITS rDNA) and their effectiveness in determinating relationships in Sellaphora (Bacillariophyta). - Protist 158: 349-364.

Finlay, B.J.; Monaghan, E.B. \& Maberly, S.C. (2002): Hypothesis: the rate and scale of dispersal of freshwater diatom species is a function of their global abundance. - Protist 153: 261-273.

Fránková, M.; Bojková, J.; PoulíčKová, A. \& Hájek, M. (2009): The structure and species richness of the diatom assemblages of the Western Carpathian spring fens along gradient of mineral richness. - Fottea 9: $355-368$.

Geitler, L. (1951): Der baudes zellkerns von Navicula radiosa und verwandten artn und die präanaphasische trennung von tochtercentromeren. - Öster. Bot. Z. 98: $206-214$.

Geitler, L. (1952a): Untersuchungen über kopulation und auxosporenbildung pennater diatomeen iii. gleichartigkeit der gonenkerne und verhalten des heterochromatins bei Navicula radiosa. - Öster. Bot. Z. 99: 469-482.

Geitler, L. (1952b): Untersuchungen über kopulation und auxosporenbildung pennater diatomeen iv. vierkernige zygoten bei Navicula cryptocephala var. veneta fa. V. Allogamie bei Synedra rumpens var. fragilarioides. - Öster. Bot. Z.: 99: 598-605.

GeITLER, L. (1958): fortpflanzungsbiologische eigentümlichkeiten von Cocconeis und vorarbeiten zu einer systematischen gliederung von Cocconeis placentula nebst beobachtungen an bastarden. - Öster. Bot. Z. 105: $350-379$. 
Guiry, M. D. \& Guiry, G. M. (2015): AlgaeBase. Worldwide electronic publication, National University of Ireland, Galway. Retrieved from http://www.algaebase.org; searched on 14 April 2015.

Hásek, F.; PoulíčKová, A.; Vašutová, M.; SyrovátKa, V.; Jiroušek, M.; ŠtepánKová, J.; Opravilová, V. \& HÁJKovÁ, P. (2014): Small ones and big ones: crosstaxon congruence reflects organism body size in ombrotrophic bogs. - Hydrobiologia 726: 95-107.

HÁJEK, M.; HeKerA, P. \& HÁJKovÁ, P. (2002): Spring fen vegetation and water chemistry in the Western Carpathian flysch zone. - Folia Geobotanica 37: 205-224.

HájKová, P.; Bojková, J.; FránKová, M.; Opravilová, V.; HájeK, M.; Kintrová, K. \& Horsák, M. (2011): Disentangling the effects of water chemistry and substrastum structure on moss-dwelling unicellular and multicellular microorganisms in spring fens. $-\mathbf{J}$. Limnol., 70, Suppl. 1: 54-64.

HiNTZ, J.L. (2007): Users's guide I. NCSS Statistical System. Kaysville, Utah. Retrived from: http://ncss.wpengine.netdna-cdn.com/wp-content/uploads/2012/09/ NCSSUG1.pdf;searched on 27 May 2015.

Hofmann, G.; Werum, M. \& Lange-Bertalot, H. (2013): Diatomeen im Süßwasser-Benthos von Mitteleuropa. Bestimmungsflora Kieselalgen für die ökologische Praxis. Über 700 der häufigsten Arten und ihre Ökologie. - 908pp., Koeltz Scientific Books, Königstein.

Hudsted, F. (1930): Bacillariophyta (Diatomeae). In Pascher, A. (Ed.) Die Süßwasser-Flora Mitteleuropas. Heft 10. Zweite Auflage. - pp. 466, Gustav Fischer, Jena.

Hustedt, F. (1985): Die Kieselalgen Deutschlands, Österreichs und der Schweiz Bd. 7, Teil 2. Leipzig.

JAHN, R.; KusBer, W.-H. \& Romero, O.E. (2009): Cocconeis pediculus Ehrenberg and C. placentula Ehrenberg var. placentula (Bacillariophyta): Typification and taxonomy. - Fottea 9: 275-288.

Jahn, R.; Zetzesche, H.; Reinhardt, R. \& Gemeinholzer, B. (2007): Diatoms and DNA barcoding: A pilot study on an environmental sample. -In: KUSBER, W.-H. \& $\mathrm{J}_{\mathrm{AHN}}, \mathrm{R}$. (eds): Proceedings of the 1st Central European Diatom Meeting.

Jeppensen, E.; Jenses, J.P.; Søndergaard, M.; Lauridsen, T. \& LANDKILDEHUS, F. (2000): Trophic structure, species richness and biodiversity in Danish lakes: changes along a phosphorus gradient. - Freshwat. Biol. 45: 201-218.

Kahlert, M.; Albert, R.-L.; Anttilla, E.-L.; Bengtsson, R.; Bigler, C.; Eskola, T. Gälman, V.; Gottschalk, S.; Herlitz, E.; Jarlman, A.; Kasperoviciene, J.; KoKOCIŃSKI, M.; LuUP, H.; MietTinen, J.; PAunksnyte, I.; Pirrsoo, K. Quintana, I.; Raunio, J.; Sandell, B.; Simonla, H.; SundBerg, I.; Vilbaste, S. \& Weckström, J. (2009): Harmonazation is more important than experience-results of the first Nordic-Baltic diatom intercalibration exercise 2007 (stream monitoring). J. Appl. Phycol. 21: 471-482.

Kelly, M. G.; King, L. \& Ni Chathain, B. (2009): The conceptual basis of ecological status assessments using diatoms. - Proc. R. Ir. Acad. 109: 175-189.

Kelly, M.G.; Cazaubon, A.; Coring, E.; Dell'uomo, A.; Ector, L.; Goldsmith, B.; Guasch, H.; HürlimanN, J.; Jarlman, A.; Kawecka, B.; Kwandrans, J.; LaUguste, R.; Lindstrøm, E.A.; Leitao, M.; Marvan, P.; PadisÁk, J.; Pipp, E.; Prygiel, J.; Rott, E.; SAbater, S.; VAn Dam, H.; Vizinet, J. (1998): Recommenda- tions for the routine sampling of diatoms for water quality assessments in Europe. - J. Appl. Phycol. 10: 215-224.

Kelly, M.G.; Kennedy, B.; Bennet, C.; Mykrä, H.; Miettin, J.; VUORI, K.-M.; KAHLERT, M. \& GÖNCZI, M. (2007): Northern rivers GIG phytobenthos intercalibration exercise. - 32pp., N. GIG Phytobenthos IC Report Technical report.

Kermarrec, L.; Rimet, F.; Chaumeil, P. Humbert, J.-F. \& Bouchez, A. (2013): Next generation sequencing to inventory taxonomic diversity in eukaryotic communities: a test for freshwater diatoms. - Mol. Ecol. Resour. 13: 607-619.

King, L.; Clarke, G. Bennion, H; Kelly, M. \& Yallop, M. (2006): Recommendations for sampling littoral diatoms in lakes for ecological status assessments. $-\mathrm{J}$. Appl. Phycol. 18: 15-25.

Kitner, M. \& PoulíčKovÁ, A. (2003): Littoral diatoms as indicators for eutrophication of shallow lakes. - Hydrobiologia 506-509: 219-524.

KovÁcs, C.; Kahlert, M. \& PAdisÁK, J. (2006): Benthic diatom communities along $\mathrm{pH}$ gradients in Hungaria and Swedish streams. - J. Appl. Phycol. 18: 105-117.

Krammer, K. \& Lange-Bertalot, H. (1986): Bacillariophyceae: 1. Teil: Naviculaceae. - In: Ettr, H.; GerlofF, J.; Heynig, H. \& Mollenhauer, D. (eds): Süßwasserflora von Mitteleuropa. - 876 pp., Gustav Fisher Verlag, Jena.

Krammer, K. \& Lange-Bertalot, H. (1988): Bacillariophyceae: 2. Teil: Epithemiaceae, Surirellaceae. - In: Ettl, H.; Gerloff, J.; Heynig, H. \& Mollenhauer, D. (eds): Süßwasserflora von Mitteleuropa - 876 pp., Gustav Fisher Verlag, Jena.

Krammer, K. \& Lange-Bertalot, H. (1991): Bacillariophyceae: 3. Teil: Centrales, Fragilariaceae, Eunotiaceae. - In: Ettr, H.; Gerloff, J.; Heynig, H. \& MollenHAUER, D. (eds): Süßwasserflora von Mitteleuropa 576 pp., Gustav Fisher Verlag, Jena.

Krammer, K. \& Lange-Bertalot, H. (1997): Baciollariophyceae, 2 (1-4). Süßwasserflora von Mitteleuropa. - 1991 pp., Fisher press, Stuttgart.

Krammer, K. \& Lange-Bertalot, H. (2004): Bacillariophyceae: 4. Teil: Achnanthaceae, Kritische Erganzungen zu Navicula (Lineolatae), Gomphonema Gesamtliteraturverzeichnis Teil 1-4. - In: EtTl, H.; GerlofF, J.; Heynig, H. \& Mollenhauer, D. (eds): Süßwasserflora von Mitteleuropa - 468 pp., Gustav Fisher Verlag, Jena.

Krammer, K. (2000): Diatoms of Europe. - In:LANGe-BertaLot, H. (ed.): The Genus Pinnularia, Vol. 1. - 703 pp., A. R. G. Gantner Verlag K. G., Ruggel.

Krammer, K. (2002): Diatoms of Europe. - In: LANGE-BertALOt, H. (ed.): The genus Cymbella, Vol. 3. -584 pp., A. R. G. Gantner Verlag K. G., Ruggel.

Krammer, K. (2003): Diatoms of Europe. - In: Lange-BertALOT H. (ed.) Cymbopleura, Delicata, Navicymbula, Gomphocymbellopsis, Afrocymbella Vol. 4, Supplements to cymbelloid taxa. - 530 pp., A. R. G. Gantner Verlag K. G., Ruggel.

Kwandrans, J.; Florantam, P.; Kawecka, B. \& Krzysztof, W. (1998): Use of benthic diatom communities to evaluate water quality in rivers of southern Poland. - J. Appl. Phycol. 10: 193-201.

Lange-Bertalot, H. (2001): Diatoms of Europe (LangeBertalot H., ed.) Volume 2: Navicula sensu stricto, 
10 Genera Separated from Navicula sensu stricto, Frustulia. -526 pp., A. R. G. Gantner Verlag K. G., Ruggel.

Lange-Bertalot, H.; BaK, M.; Witkowski, A. \& TagliavenTI, N. (2011): Diatoms of Europe. - In: LANGE-BERTALOT, H. (ed.): Eunotia and some related genera, Vol. 6. - 747 pp., A. R. G. Gantner Verlag K. G., Ruggel.

Lavoie, I.; Somers, K.M.; Paterson, A. M. \& Dillon, P. J. (2005): Assessing scales of variability in benthic diatom community structure. - J. Appl. Phycol.17: 509-513.

Mann, D.J.; Mcdonald, S.M.; BAYer, M.M.; DroOP, S.J.M.; Chepurnov, V.A.; LoKe, R.E.; Ciobanu, A. \& Du Buf, J.M.H. (2004): The Sellaphora pupula species complex (Bacillariophyceae): morphometric analysis, ultrastructure and mating data provide evindence for five new species. - Phycol. 43: 459-482.

Mann, G.M.; Thomas, S.J. \& Evans, K.M. (2008): Revision of the diatom genus Sellaphora: a first account of the larger species in the British Isles. - Fottea 8: 15-78.

Morales, E.A.; Guerrero, J.M.; Wetzel, C.E.; SAla, S. \& ECTOR LuC (2013): Unraveling the identity of Fragilaria pinnata Ehrenberg and Staurosira pinnata Ehrenberg: research in progress on a convolutes story. - Cryptogamie Algol. 34: 89-102.

Patrick, R. \& Reimer, C.W. (1975): The diatoms of the United States, Exclusive of Alaska and Hawaii, Volume 2, Part 1 - Entomoneidaceae, Cymbellaceae, Gomphonemaceae, Epithemaceae. -213 pp. Academy of Natural Sciences of Philadephia Monograph.

Ponander, K.C. \& Potapova, M.G. (2007): Diatoms from the genus Achnanthidium in flowing waters of the Appalachian Mountains (North America): Ecology, distribution and taxonomic notes. - Limnologica 37: 227-241.

Potapova, M. \& Hamilton, P.B. (2007): Morphological and ecological variation within the Achnanthidium minutissimum (Bacillaryophyceae) species complex. - J. Phycol. 43: 561-575.

Potapova, M. \& Charles, D.F. (2003): Distribution of benthic diatoms in U.S. rivers in relation to conductivity and ionic composition. - Freshwater Biol. 48: 1311-1328.

Potapova, M. \& Charles, D.F. (2007): Diatom metrics for monitoring eutrophication in rivers of the United States. - Ecol. Indic. 7: 48-70.

PoulíčKová, A.; Duchoslav, M. \& Dokulit, M. (2004): Litoral diatom assemblages and bioindicators of lake trophic status. A case study from perialpine lakes in Austria. Eur. - J. Phycol. 39: 143-152.

Poulíčnová, A.; Dvořák, P.; Mazalová, P. \& Hašler, P. (2014): Epipelic microphotographs: an overlooked assemblage in lake ecosystems. - Freshw. Sci. 33: 513-523.

PoulíčKová, A.; HáJKová P., KŘenková P. \& HájeK M. (2004): Distribution of diatoms and bryophytes on linear transects through spring fens. - Nova Hedwigia 78: 411-424.

PoulíčKová, A.; Hašler, P.; Lysáková, M. \& Spears, B. (2008): The ecology of freshwater epipelic algae: an update. - Phycol. 47: 437-450.

PoulíčKovÁ, A.; ŠpačKová, J.; Kelly, M. G. \& Mann, D.G. (2008): Ecological variation within Sellaphora species complexes (Bacillariophyceae): specialists or genealists? - Hydrobiologia 614: 373-386.
PoulíčKovÁ, A.; Veselá, J.; Neustupa, J. \& ŠKaloud, P. (2010): Pseudocryptic diversity versus cosmopolitanism in diatoms: a case study Navicula cryptocephala Kütz. (Bacillariophyceae) and morfologically similar taxa. - Protist 161: 353-369.

Prygiel, J.; Carpentier, P.; Almeida, S.; Coste, M.; Druart, J.C.; ECtor, L.; Guillard, D.; Honoré, M.A.; Iserentant, R.; Ledeganck, P.; Lalanne-Cassou, Ch.; Lesniak, Ch.; Merciers, I.; Moncaut, P.; Nazart, M.; Nouchet, N.; Peres, F.; Peeters, V.; Rimet, F.; Rumeau, A.; Sabater, S.; Straub, F.; Mariacristina, T.; Tudesque, L.; Van De ViJver, B.; Vidal, H.; VIZINET, J. \& ZYDEK, N. (2002): Determination of the biological diatom index (IBD NF T 90-354): results of an intercomparison exercise. - J. Appl. Phycol.14: $27-39$.

RAPANT, S.; VRÁnA, K \& Bodiš, D. (1996): Geochemical Atlas of Slovakia. Part Groundwater. GSSR, Bratislava.

Rimet, F. \& Bouchez, A. (2012): Life-forms, cell-sizes and ecological guilds of diatoms in European rivers. Knowl. Manag. Aquat. Ecosyst. 406, 01.

SigEe, D. (2005): Freshwater mikrobiology: Biodiversity and dynamic interactions of microorganisms in the aquatic environment. University of Manchester, N.J.: Wiley \& Sons, Ltd. Retriever from: http://samples. sainsburysebooks.co.uk/9780470026472_sample_384565.pdf; searched on 14th May 2015.

ŠPačKovÁ, J.; Hašler, P.; ŠTĚPÁnková, J. \& PoulíčKovÁ, A. (2009): Seasonal succession of epipelic algae: a case study on a mesotrophic pond in a temperate climate.Fottea 9: 121-133.

Taylor, J.C.; Harding, W.R. \& Archiblad, C.G.M. (2007): A methods manual for collection, preparation and analysis of diatom samples version 1.0. - WRC Report TT 281/07. Retriever from: http://docs.niwa.co.nz/ library/public/1770054839.pdf; searched on 14 May 2015.

Ter Braak, C.J.F. \& Šmilauer, P. (2002): CANOCO reference manual and CanoDraw for Windows user's guide: Software for Canonical Community Ordination (version 4.5). - 500 pp., Ithaca, NY, Microcomputer Power.

Tolasz, R.; Míková, T.; Valeriánová, A. \& Voženílek, V. (2007): Atlas podnebí Česka. - 255pp., Univerzita Palackého v Olomouci. ČHMU.

Trobajo, R.; Clavero, E.; Chepurnov, V.A.; Sabbe, K.; Mann, D.G.; IshiHara, S. \& Cox, E.J. (2009): Morphological, genetic and mating diversity within the widespread bioindicator Nitzschia palea (Bacillariophyceae). - Phycologia 48: 443-459.

Van Der Vijver, B.; Wetzel, C.; Kopalová, K.; Zidarova, R. \& EстоR, L. (2013): Analysis of the type material of Achnanthidium lancolatum Brébisson ex Kützing (Bacillariophyta) with the description of two new Planothidium species from Antarctic Region. Fottea 13: 105-117.

Vanormelingen, P.; Evans, K.M.; Chepurnos, V.A.; VyverMAN, W. \& MANN, D.G. (2013): Molecular species discovery in the diatom Sellaphora and its congruence with mating trials. - Fottea 13: 133-148.

Vanormelingen, P.; Chepurnov, V. A.; Mann, D.G.; Sabbe, K. Vyverman, W. (2008): Genetic divergence and reproductive barriers among morphologically heterogeneous sympatric clones of Eunotia bilunaris Sensu lato (Bacillariophyta). - Protist 159: 73-90. 
Wang, P.; Park, B.S.; Kim, J.H., Kim, J.-H.; LeE, H.O. \& HAN, M.S. (2014): Phylogenetic position of eight Amphora sensu lato (Bacillariophyceae) species and comparative analysis of mophological characteristics. - Algae 29: 57-73.

Wetzel, C.E.; Ector, L.; Van de Vijver, B.; Compére, P. \& Mann, D.G. (2015): Morphology, typification and critical analysis of some ecologically important small naviculoid species (Bacillariophyta). - Fottea 15: 203-234

Williams, D.M. (2011): Synedra, Ulnaria: definitions and descriptions - a partial resolution. - Diatom Res. 26: 149-153.

YANG, H. \& Flower, R.J. (2012): Effects of light and substrate on the benthic diatoms in an oligotrophic lake: a comparison between antural and artificial substrates. - J. Phycol. 48: 1166-1177.

(C) Czech Phycological Society (2015)

Received April 1, 2015

Accepted July 20, 2015 